\title{
MONOSTOTIC OSTEOFIBROUS DYSPLASIA OF THE TIBIA
}

\author{
Maimoona Siddique ${ }^{1}$, Humayun Bashir ${ }^{1}$, Imran Khalid Niazi ${ }^{2}$, Ahsan Shamim ${ }^{3}$ \\ ${ }^{1}$ Department of Nuclear Medicine, Shaukat Khanum Memorial Cancer Hospital and Research Centre, Lahore, \\ Pakistan, ${ }^{2}$ Department of Radiology, Shaukat Khanum Memorial Cancer Hospital and Research Centre, Lahore, \\ Pakistan, ${ }^{3}$ Department of Surgical Oncology, Shaukat Khanum Memorial Cancer Hospital and Research Centre, \\ Lahore, Pakistan
}

Received: 15 March 2016 / Accepted: 8 April 2016

\begin{abstract}
An 11-year-old boy presented with painful swelling of leg following fracture of tibia. Osteofibrous dysplasia (OFD) is an uncommon, benign, non-hereditary bone disorder in which fibrous tissue develops in place of normal bone that affects the young adults in their first and second decade of life. Tc-99m MDP whole-body bone scintigraphy revealed increased tracer uptake in dense proximal tibia. On single-photon emission computed tomography-computed tomography (SPECT-CT), radiological features of cortical-based lesion superimposed on abnormal tracer uptake confirmed it to be a monostotic OFD. This case emphasises the role of SPECT-CT and magnetic resonance imaging in detecting osteofibrous dysplasia and differentiating it from other benign bone conditions.
\end{abstract}

Key words: Bone scintigraphy, magnetic resonance imaging, monostotic osteofibrous dysplasia, single-photon emission computed tomography-computed tomography, tibia

\section{Introduction}

Osteofibrous dysplasia (OFD) is a rare, asymptomatic, non-neoplastic condition of unknown origin that affects the long bones. ${ }^{[1]}$ It has been postulated to arise from a fibrovascular defect. It has been closely associated with adamantinomas. According to one theory, OFD results from an abnormality in the Haversian canals, whereas adamantinoma develops secondary to a defect of intramedullary vasculature. ${ }^{[2]}$ OFD was first described by Frangenheim, in 1921, and reported it as a congenital osteitis fibrosa. ${ }^{[3]}$ Subsequently, many authors described this lesion in literature, and finally, Campanacci and Laus devised the term OFD of the tibia, replacing ossifying fibroma due to its congenital origin, the histologic resemblance to fibrous dysplasia and the dominant involvement of the tibia and fibula. OFD is also referred to as Campanacci syndrome. ${ }^{[4]}$

OFD is a slow-growing cortical-based lesion. In contrary to fibrous dysplasia that is a medullary cavity-based lesion and mainly affects the craniofacial skeleton, long bones

Correspondence: Dr. Maimoona Siddique, Department of Nuclear Medicine, Shaukat Khanum Memorial Cancer Hospital and Research Centre, Lahore, Pakistan.

Email: maimoona_kashif@hotmail.com and ribs, ${ }^{[5]}$ OFD mainly affects the tibia or fibula and in few cases, radius or ulna. ${ }^{[6]}$ The tibial involvement was found in 35 cases reported by Campanacci and Laus; four cases had ipsilateral involvement of the fibula (11\%) and 22 lesions $(63 \%)$ affected the middle third of the tibial diaphysis. ${ }^{[7]}$ Ishida et al. reported 11 lesions (92\%) in the proximal tibial diaphysis, with one lesion in the ulna. ${ }^{[8]}$

OFD is usually diagnosed in children younger than 10 years, with a peak incidence in children aged $1-5$ years. Several newborn cases have also been reported. ${ }^{[9]}$ Since OFD is a rare disease, there are limited data in literature about characteristics of disease and response to medical treatment. There is no spontaneous resolution of OFD. Nonsurgical treatment is recommended until skeletal maturity is reached. Recurrent pathologic fractures may be an ongoing problem in some active children. Using a tibial brace similar to those used for congenital pseudarthrosis of the tibia may minimise recurrent pathologic fractures. Surgery should be performed if complications are imminent, such as marked bone deformation or pseudoarthrosis. ${ }^{[10]}$

\section{Case Report}

An 11-year-old male boy presented with a painful, progressively increasing swelling of the left tibia for a 
few months. Preceding this complaint, there was a history of fracture at same site 4 years back while playing and was treated with cast. No associated history of weight loss, fever, or night sweats. On examination, there was a tender swelling involving the extensor surface of the left proximal leg with no signs of inflammation. Distal neurovascular examination was unremarkable. Threephase Tc-99m whole-body bone scintigraphy [Figure 1] revealed hyperemia, increased blood pool activity and abnormal intense tracer uptake involving proximal $1 / 3$ of diaphysis of the left tibia. In addition, subtle focal tracer uptake was seen in the mid diaphysis of the left tibia. On single-photon emission computed tomography-computed tomography (SPECT-CT) images [Figure 2], there was a corresponding expansile, predominantly lytic, cortical-

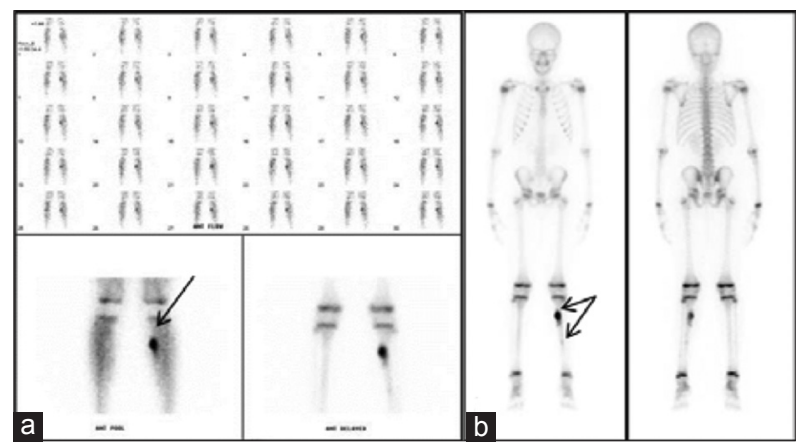

Figure 1: Three-phase bone scintigraphy (a) showed hyperemia and increased blood pool activity in the proximal left leg (black arrows). Delayed static images (b) show hot spots in the left tibial diaphysis caused by abnormal increased uptake of the radioisotope technetium-99m methylene diphosphonate ( ${ }^{99 \mathrm{~m}} \mathrm{Tc} \mathrm{MDP}$ )

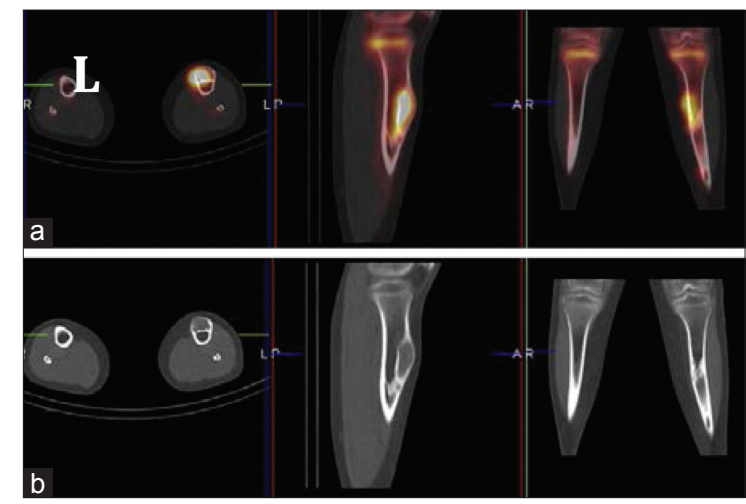

Figure 2: Single-photon emission computed tomography/ computed tomography images showed MDP avid expansile cortical lesions involving the left proximal tibial diaphysis with central ground glass appearance and peripheral intact zone of cortical thickening based "ground glass" appearance involving proximal left tibial diaphysis with peripheral zone of sclerosis and cortical thickening. There was no associated soft tissue component. A greenstick pathological fracture line was also seen adjacent to this lesion. Faint tracer uptake in midtibia showed underlying lucencies with peripheral sclerosis. Further, correlative radiological imaging (X-ray [Figure 3] and magnetic resonance imaging [MRI] [Figure 4]) supported the diagnosis of fibrous dysplasia.

\section{Discussion}

OFD is an unusual developmental condition of childhood, which mainly affects the tibial diaphysis. It follows a

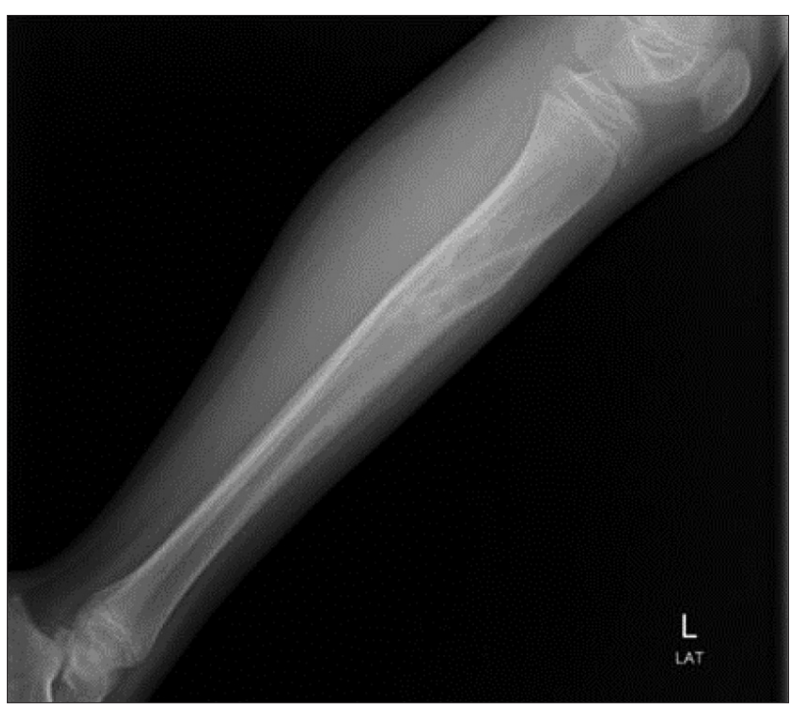

Figure 3: Craniocaudal view; ill-defined radiopaque mass in the left retroareolar region, protruding on skin

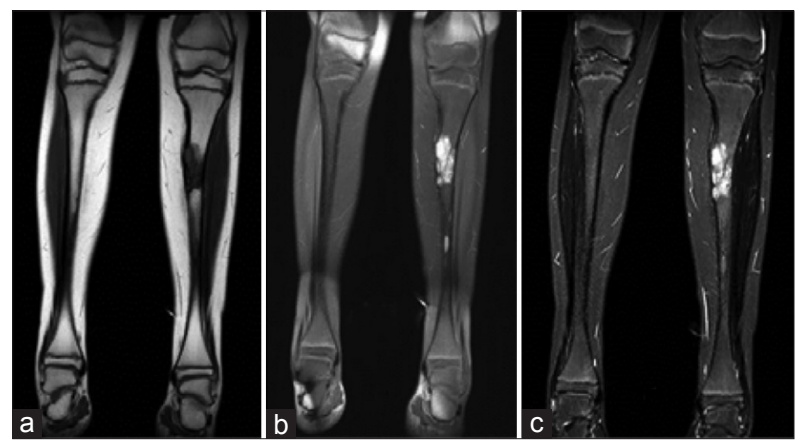

Figure 4: Magnetic resonance imaging shows typical features with low-to-intermediate signal intensity equal to that of muscle on T1-weighted image (a). Nodular enhancement pattern is seen on post-gadolinium contrast enhancement sequence (b). No surrounding medullary oedema is seen on STIR contrast image (c) 
slowly progressive course and tends to stabilise after skeletal maturity. The possible link with adamantinoma still remains controversial and some authors believe that they are a spectrum of same histological process. ${ }^{[1,2]}$ Histologically, OFD is characterised by an osteofibrous stroma replacing the normal bone, showing trabecular pattern which, unlike in FD, is surrounded by a regular rim of prominent cubic osteoblasts, mature lamellar bone and zonal segmentation. Radiologic features are eccentric, lytic lesions, usually involving tibial diaphysis with 'ground glass' appearance. Differential diagnosis includes FD and adamantinoma. Just like OFD, the latter occurs predominantly in the tibia, its malignant potential remains high, calling for radical surgical treatment. According to literature review, the mean age for OFD was 9 years, which is considerably lower than that of patients with FD (30 years). The lesions were found exclusively in the tibia. Unlike in FD, once bone maturation is completed, surgical treatment of OFD is not normally followed by recurrent disease. Thus, the prognosis of OFD with restricted surgical therapy is more favourable than that of FD. ${ }^{[11,12]}$

Scintigraphically, OFD lesions show increased uptake due to high bone turnover. Although BS is sensitive for OFD, it lacks specificity. The poor specificity is improved with the use of SPECT-CT which concomitantly characterises the metabolic and anatomic features of the disease. ${ }^{[13]}$ The differentiating feature from malignant conditions, like adamantinomas, is the absence of soft tissue component with a well-preserved cortical margin. ${ }^{[14]}$ Very few case reports are published regarding the use of bone SPECT-CT in the detection of OFD. ${ }^{[15]}$ Radiologic correlative imaging such as MRI and X-rays is further helpful in characterisation and diagnosis.

\section{Conflict of Interest}

The authors declare that they have no conflict of interest.

\section{References}

1. McCaffrey M, Letts M, Carpenter B, et al. Osteofibrous dysplasia: A review of the literature and presentation of an additional 3 cases. Am J Orthop 2003;32:479-86.

2. Gleason BC, Liegl-Atzwanger B, Kozakewich HP, et al. Osteofibrous dysplasia and adamantinoma in children and adolescents: A clinicopathologic reappraisal. Am J Surg Pathol 2008;32:363-76.

3. Frangenheim P. Congenital ostitis fibrosa as the cause an intrauterine fracture of the lower leg. Arch Klin Chir 1921;117:22-9.

4. Campanacci M. Osteofibrous dysplasia of long bones a new clinical entity. Ital J Orthop Traumatol 1976;2:221-37.

5. Feller L, Wood NH, Khammissa RA, et al. The nature of fibrous dysplasia. Head Face Med 2009;5:22.

6. Davidson D, Chapurlat G. Osteofibrous Dysplasia. Available from: http://www emedicine.medscape.com/ article/1256595-overview. [Last accessed on 2016 Mar 11].

7. Campanacci M, Laus M. Osteofibrous dysplasia of the tibia and fibula. J Bone Joint Surg Am 1981;63:367-75.

8. Ishida T, Iijima T, Kikuchi F, et al. A clinicopathological and immunohistochemical study of osteofibrous dysplasia, differentiated adamantinoma, and adamantinoma of long bones. Skeletal Radiol 1992;21:493-502.

9. Zamzam MM. Congenital osteofibrous dysplasia of the tibia, associated with pseudoarthrosis of the ipsilateral fibula. Saudi Med J 2008;29:1507-9.

10. Lee RS, Weitzel S, Eastwood DM, et al. Osteofibrous dysplasia of the tibia. Is there a need for a radical surgical approach? J Bone Joint Surg 2006;88:658-64.

11. Bosse A, Niesert W, Wuisman P, et al. Fibrous dysplasia versus osteofibrous dysplasia. Morphological, differential diagnostic and clinical aspects. Experiences from the Westfalen bone tumor registry. Z Orthop Ihre Grenzgeb 1993; 131:42-50.

12. DiCaprio MR, Enneking WF. Fibrous dysplasia. Pathophysiology, evaluation, and treatment. J Bone Joint Surg Am 2005;87:1848-64.

13. Wang K, Allen L, Fung E, et al. Bone scintigraphy in common tumors with osteolytic components. Clin Nucl Med 2005;30:655-71.

14. Tuncel M, Kiratli PO, Gedikoglu G. SPECT CT imaging of poliostotic fibrous dysplasia. Rev Esp Ned Nucl Imagen Mol 2012;31:47-8.

15. Nakahara T, Fujii H, Hashimoto J, et al. Use of bone SPECT in the evaluation of fibrous dysplasia of the skull. Clin Nucl Med 2004;29:554-9. 